\title{
Estudo da variabilidade do NDVI sobre o Brasil, utilizando-se a análise de agrupamentos
}

\author{
Helen da C. Gurgel ${ }^{1}$, Nelson J. Ferreira² \& Alfredo J. B. Luiz ${ }^{3}$ \\ 1 DSR/INPE. CP 515, CEP 12.201-970, São José dos Campos, SP. Fone: (12) 3945-6643. E-mail: helen.gurgel@ig.com.br (Foto) \\ 2 DSR/INPE, E-mail: nelson@ltid.inpe.br \\ ${ }^{3}$ Embrapa Meio Ambiente. Rodovia SP340, Campinas/Mogi Mirim, km 1275, CEP 13820-000, Jaguariúna, SP. \\ Fone: (19) 3867-8700. E-mail: alfredo@cnpma.embrapa.br
}

Protocolo 60 - 29/4/2002 - Aprovado em 15/4/2003

\begin{abstract}
Resumo: Este trabalho analisa a variabilidade do NDVI (Índice de Vegetação por Diferença Normalizada) sobre o Brasil, utilizando-se a análise de agrupamentos. As análises foram feitas através de imagens do sensor Advanced Very High Resolution Radiometer (AVHRR) para o período de janeiro de 1982 a dezembro de 1993. Os resultados obtidos mostram que na região Amazônica o ciclo anual do NDVI não é bem definido, visto que o máximo tipicamente ocorre em junho, dois meses após o período chuvoso, enquanto o mínimo se dá em dois períodos distintos: entre fevereiro e março e setembro e novembro. Na região central do Brasil, o cerrado apresenta um ciclo anual definido, com valores máximos de NDVI entre março e maio, e mínimos em setembro, final do período seco. Por outro lado, a vegetação das regiões da zona da mata nordestina e dos campos de Roraima apresentam um ciclo anual nítido, sendo que os maiores valores de NDVI ocorrem em junho e julho e os menores entre fevereiro e março, alguns meses antes do início das chuvas. No caso da região nordeste do Brasil, a caatinga mostra um ciclo anual bem definido, com um período seco marcante, sendo que os valores mais elevados de NDVI ocorrem entre os meses de abril e maio, que é o final da época das chuvas, e os menores em setembro e outubro. Em parte do Estado de Santa Catarina e no sul do Paraná, o ciclo anual das formações vegetais dominantes (floresta ombrófila aberta e floresta ombrófila mista) não é muito nítido. No sul do Brasil, a região de estepes não apresenta ciclo anual nítido, os valores máximos de NDVI geralmente ocorrem entre março e junho e os valores mínimos no mês de agosto. Além disso, constatou-se que os eventos El Niño, independente da sua intensidade, afetam distintamente os vários tipos de vegetação.
\end{abstract}

Palavras-chave: cobertura vegetal, El Niño, clima

\section{Study of NDVI variability in Brazil using cluster analysis}

Abstract: This paper deals with vegetation cover variability in Brazil using cluster analysis. The study was done using NDVI (Normalized Difference Vegetation Index) images from the AVHRR (Advanced Very High Resolution Radiometer) sensors for the January1982-December1993 period. The results show that the annual cycle of NDVI in the Amazon region is not well defined; the maximum values typically occur in June, two months after the rainy season, while the minimum ones occur in two distinct periods: February-March and September-November. In Central Brazil, the Savannas has a well defined annual cycle, showing maximum NDVI values around March and May and a minimum in September. On the other hand, the seasonal variability of the Northeast Brazil (NE) "Zona da Mata" (Atlantic Forest) and Savannas of Roraima vegetation cover show high NDVI values in June and July and low values between February and March, a few months before the rainy season onset. In the case of NE, the "Caatinga" (thorn shrub) shows a well defined annual cycle with a remarkable dry period, the highest NDVI values occur between April and May, which is the end of the rainy season, and the smallest values occur in September and October. In portions of Santa Catarina and southern part of Parana State, the annual cycle of the prevailing vegetation cover (open ombrophylous forest and mixed ombrophylous forest) is not well defined, while in Southern Brazil, the Steppe region does show a seasonal variability, with maximum NDVI values between March and June and a minimum one in August. Also, it was observed that ENSO events, independent of their intensity, do affect the different types of vegetation cover mainly the dense and greener forest types (e.g. the Amazon forest).

Key words: vegetation cover, El Niño, climate 


\section{INTRODUÇÃO}

Nas últimas décadas, as mudanças provocadas pelo homem no meio ambiente têm sido intensas, ocasionando impactos de diferentes naturezas no solo, na água, na atmosfera, na biodiversidade e na população humana. A conscientização dessas mudanças cresceu e os estudos sobre mudanças globais e seus impactos têm sido destacados no campo das ciências da natureza.

Recentemente, atenção considerável tem sido dada aos índices de vegetação calculados a partir do sensor da série Advanced Very High Resolution Radiometer (AVHRR) National Oceanic and Atmospheric Administration (NOAA). Esses índices têm sido amplamente usados para o monitoramento regional ou global da cobertura vegetal (e.g. Eastman \& Fulk 1993, Anyamba \& Eastman 1996; Potter \& Brooks 1998; Richard \& Poccard 1998).

No Brasil, vários estudos têm demonstrado que o NDVI se correlaciona com algumas variáveis climáticas em amplas condições ambientais. Liu et al. (1991) utilizaram dados de NDVI obtidos de imagens AVHRR/NOAA no modo Global Area Coverage (GAC) no período de junho de 1981 a agosto 1987 , sobre a América do Sul. A evolução mensal do NDVI para anos secos e úmidos do período de estudo, foi utilizada para se estimar as áreas com cobertura de NDVI, menores que 0,23. Este valor foi usado como indicador de áreas secas e como delimitador de áreas de baixa precipitação no continente. Eles verificaram, ainda, que na maioria das regiões a maior correlação entre o NDVI e a quantidade de precipitação ocorre quando se compara NDVI com a precipitação do mês anterior. Esse estudo mostrou, também, que a distribuição continental de vegetação com estresse, indicada pelo NDVI, corresponde a áreas que sofreram impactos de seca provocados pelo El Niño. Batista et al. (1997) analisaram a composição mensal do NDVI (AVHRR/ NOAA) da região amazônica, para o período de agosto de 1981 a junho de 1991. Eles averiguaram que os tipos de vegetação dominantes podem ser diferenciados e que é possível identificar-se variações da cobertura vegetal associadas a mudanças climáticas. As composições mensais de NDVI revelaram uma forte sazonalidade no cerrado, especialmente na caatinga, enquanto os demais tipos de cobertura florestal mostraram pequena variação ao longo dos anos. As composições anuais de NDVI indicaram uma clara e significante redução nos anos secos, associada ao evento ENSO. Barbosa (1998) avaliou composições mensais do período de 1982 a 1985 de NDVI provenientes das imagens AVHRR/NOAA e totais mensais de precipitação do Nordeste do Brasil. Ele mostrou que as distribuições espacial e temporal do NDVI e a precipitação mensal na região têm padrões semelhantes e são estatisticamente correlacionados. Desta forma, o NDVI poderia ser utilizado como indicador de regime pluviométrico no Nordeste do País.

Recentemente, Gurgel (2000) analisou as conexões entre o NDVI e as variabilidades climáticas anual e interanual sobre o Brasil, aplicando a técnica de análise por componentes principais (ACP) aos dados mensais de NDVI derivado do AVHRR, para o período de janeiro de 1982 a dezembro de 1993, cujos resultados mostram que a ACP aplicada ao NDVI possibilita caracterizar a variabilidade anual e interanual dessas tipologias de vegetação relacionadas com a variabilidade climática (componentes 1, 2 e 3). A ACP também revelou os principais fatores "não-climáticos", tais como ocorrência de queimadas e erros de interpretação, devido à mudança de horário da passagem dos satélites NOAA e troca dos satélites NOAA 9 e 11 (componentes 4, 5, 6 e 10) que ocasionaram distúrbios no NDVI.

Apesar dos avanços na compreensão do NDVI no Brasil, estudos abordando a identificação e o comportamento de grupos similares dessa variável na cobertura vegetal ainda não foram realizados. O conhecimento da variabilidade espacial e temporal desses grupos de vegetação, além do interesse científico, beneficia diversos setores produtivos de nossa sociedade. Neste contexto, o principal objetivo deste trabalho foi o de avaliar a variabilidade de NDVI no Brasil, utilizando-se a análise por agrupamentos. Desta forma, espera-se localizar, no espaço geográfico, as grandes formações vegetais brasileiras, caracterizar o comportamento temporal do NDVI dessas formações e identificar os locais onde os efeitos de anomalias climáticas são mais evidentes.

\section{MATERIAL E MÉTODOS}

\section{Dados}

A análise de agrupamento foi realizada no conjunto de dados de NDVI, produzido pelo Grupo de Estudos de Monitoramento e Modelamento do Inventário Global (GIMMS - Global Inventory Modeling and Monitoring Study) da Goddard Space Flight Center/National Aeronautics \& Space Administration (GSFC/NASA). Essas imagens são composições mensais, disponibilizadas com resolução espacial de $8 \times 8 \mathrm{~km}$, na projeção "Good Interrupted Homolosine", em formato binário de 8 bits e abrange o período de janeiro de 1982 a dezembro de 1993, totalizando 144 imagens. Os dados cobrem toda a América do Sul e as informações relativas aos oceanos e aos grandes corpos d'água foram mascaradas com valor unitário. Informações completas sobre esses dados podem ser encontradas em Goddard (1999). As análises foram feitas utilizando-se uma janela de $6^{\circ} \mathrm{N}$ a $33^{\circ} 30^{\prime} \mathrm{S}$ e $34^{\circ} 30^{\prime} \mathrm{W}$ a $74^{\circ} 30^{\prime} \mathrm{W}$ sobre a América do Sul, na faixa compreendida entre os extremos norte/sul e leste/oeste do Brasil, porém, devido ao tamanho da área de estudo e ao grande número de imagens, foi necessário fazer-se uma reamostragem do tamanho do pixel, passando-o de $8 \mathrm{~km}$ para $64 \mathrm{~km}$, a fim de viabilizar a análise.

\section{Análise de agrupamento}

A técnica de classificação que mais se adequou ao objetivo de se identificar regiões semelhantes quanto ao índice de vegetação foi a análise de agrupamentos, na qual se utilizou a distância euclidiana como medida de proximidade, justificável ao se considerar que cada elemento de cena (ou pixel) de $64 \mathrm{x}$ $64 \mathrm{~km}$ constitui um objeto e que os valores de NDVI compõem os atributos quantitativos de cada um desses objetos, razão por que se obtém uma massa de dados não passível de análise humana direta. Na realidade, esse volume de dados é tão grande que limita as possibilidades de escolha entre os inúmeros métodos e algoritmos disponíveis para se realizar a tarefa de 
classificar objetos em grupo. Para atingir este objetivo, utilizouse um algoritmo de agrupamento (clustering) chamado Fastclus, que se baseia na distância euclidiana, do aplicativo SAS (SAS, 1988), em que a distância entre dois pixel, $\mathrm{x}_{1}$ e $\mathrm{x}_{2}$, é dada por:

$$
\begin{aligned}
\mathrm{d}\left(\mathrm{x}_{1}, \mathrm{x}_{2}\right) & =\left\|\mathrm{x}_{1}-\mathrm{x}_{2}\right\|=\left\{\left(\mathrm{x}_{1}-\mathrm{x}_{2}\right)^{\mathrm{t}}\left(\mathrm{x}_{1}-\mathrm{x}_{2}\right)\right\}^{\mathrm{t} / 2} \\
& =\left\{\sum_{\mathrm{i}=1}^{\mathrm{N}}\left(\mathrm{x}_{\mathrm{1}_{\mathrm{i}}}-\mathrm{x}_{2_{\mathrm{i}}}\right)^{2}\right\}^{1 / 2}
\end{aligned}
$$

em que N é o número de imagens (Richards, 1986).

Em um arquivo digital no formato ASCII, os dados foram arranjados na forma de uma matriz, na qual as linhas corresponderam aos elementos de cena (pixels) e as colunas aos valores de NDVI para cada um dos 144 meses considerados. Adicionalmente, foram levados em consideração os valores de latitude e longitude do centro de cada elemento de cena, normalizados para uma distribuição com parâmetros mais próximos daqueles encontrados nos dados de NDVI (média = 0,553 e desvio padrão $=0,162$ ) compondo, assim, mais duas colunas de atributos. Os dados deste arquivo alimentaram o algoritmo escolhido, para o qual também foram fornecidos os parâmetros: número máximo de iterações $=50$; valor do critério de convergência $=0,05$, e número máximo de grupos $=9$. Estabeleceu-se, ainda, que a escolha dos centros iniciais dos grupos seria feita de forma aleatória.

Definidos os parâmetros exigidos pelo algoritmo, o processo se inicia de forma aleatória com a definição de nove pixels, que servirão como centro dos nove grupos; a seguir, os demais pixels são divididos entre os grupos, de forma que cada um faça parte do grupo cujo centro esteja mais próximo dele empregando-se, para isto e como medida de proximidade, a distância euclidiana no espaço de atributos entre pixel e centro de grupo. Classificados todos os pixels, é calculado o valor do novo centro de cada grupo, que nada mais é que um ponto cujas coordenadas equivalem à média dos valores, para cada atributo, dos pixels daquele grupo. A medida usada como critério de convergência neste algoritmo é o valor máximo entre as distâncias dos novos centros dos grupos aos centros dos seus antecessores. As iterações param quando a mudança máxima do centro do grupo é menor ou igual ao valor fornecido como critério de convergência $(0,05$, neste caso). Como, dependendo do conjunto de dados, o critério de convergência pode exigir excessivas iterações para ser alcançado, estabeleceu-se também um número máximo de iterações, após o qual, mesmo sem atingir o critério de convergência, o procedimento é interrompido e o resultado apresentado. No caso presente limitou-se o número de iterações em 50, o que não foi atingido, pois o critério de convergência foi satisfeito com 25 iterações.

Uma vez classificados todos os elementos, calcularam-se então as médias de NDVI por grupo, de janeiro de 1982 a dezembro de 1993, e se obtiveram as médias de cada mês, considerando-se o período de doze anos, e a média anual, sempre por grupo. Esses cálculos foram também realizados com o uso do aplicativo SAS.

\section{RESULTADOS E DISCUSSÃO}

A descrição da variabilidade da precipitação utilizada para a compreensão dos resultados da análise de agrupamento, realizada nos dados de NDVI, foi baseada em Rao \& Hada (1990). A análise dos resultados obtidos na análise de agrupamento baseou-se na interpretação dos nove grupos, comparando-os e os relacionando a um ou mais tipos de vegetação, com base no mapa de vegetação do Brasil (IBGE, 1993) (Figura 1). As análises de cada grupo são apresentadas nos tópicos a seguir:

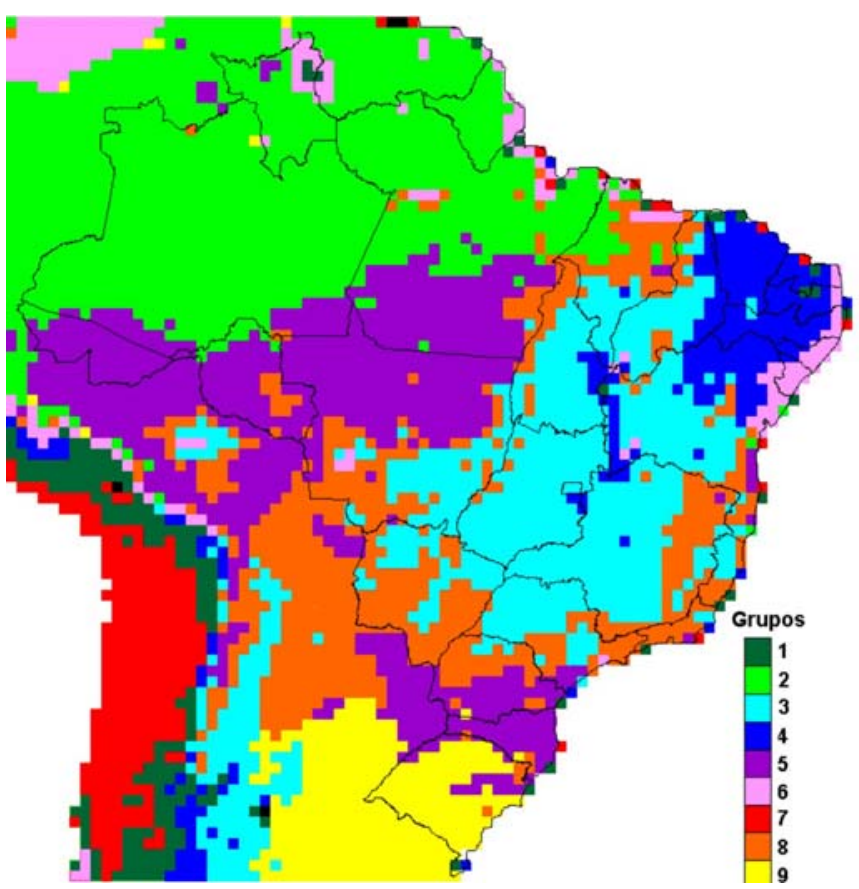

Figura 1. Classificação da cobertura vegetal sobre o Brasil, utilizando-se a análise de agrupamento e dados de NDVI

\section{Primeiro grupo}

Como a área de estudo engloba o entorno do Brasil, o primeiro grupo (verde escuro), encontrado pela análise de agrupamento foi a região dos Andes. Apesar de pequenas áreas do Brasil que possuem baixos valores de NDVI $( \pm 0,30)$ terem sido classificadas neste grupo (nordeste de Roraima, alguns pontos do litoral e da região da caatinga) ele não foi analisado, pois se situa fora da área de estudo.

\section{Segundo grupo}

O segundo grupo (verde claro) representa a região da floresta ombrófila densa (Figura 1), do norte do Brasil. Devido à pequena resolução espacial dos dados (64 x 64 km) algumas áreas de transição de floresta no noroeste do Amazonas foram classificadas neste grupo. Como se observa na Figura 2A, este tipo de vegetação não apresenta ciclo anual muito nítido. $\mathrm{O}$ máximo de NDVI dessa formação vegetal ocorre geralmente em junho, dois meses após o período de chuvas, que vai de fevereiro a abril. Almeida \& Batista (1998), avaliando este tipo de formação vegetal, também encontraram o mesmo tempo de resposta. Uma outra característica observada é a ocorrência de 
dois períodos de NDVI baixo, um entre fevereiro e março e outro entre setembro e novembro. O primeiro período, que aparentemente é mais marcante, deve ter sido influenciado pela contaminação de nuvens, muito freqüentes nesta época do ano em praticamente toda a região amazônica. Este efeito também foi detectado por Batista et al. (1993) analisando o NDVI desta região; enfim, o segundo período está relacionado à época de diminuição das chuvas na região, que se estende de julho a outubro.

$\mathrm{Na}$ Figura 2A observa-se a ocorrência de duas quedas acentuadas no NDVI nos anos de 1982/83 e 1991/92, que são anos de El Niño. No segundo caso, a queda foi mais marcante que na primeira, porém o El Niño deste período foi menos intenso que o de 1982/83. Deve-se destacar que, neste período, houve a erupção do vulcão Pinatubo (Filipinas, junho de 1991) que pode ter ocasionado diminuição do NDVI (Schultz \& Halpert, 1995). Entre 1986/87, também ocorreu o El Niño, porém o NDVI não registrou queda significativa; contudo, neste período os valores de NDVI durante a época chuvosa, ficaram abaixo da média.

\section{Terceiro grupo}

O terceiro grupo (azul claro) representa o Cerrado (Figura 1), formação vegetal encontrada na região central do Brasil. Esse tipo de vegetação apresenta ciclo anual de NDVI bastante nítido (Figura 2B), com valores máximos entre março e maio, os quais estão relacionados com o período de chuvas. Os valores mínimos são bem definidos e ocorrem no mês de setembro, final do período seco dessa região. A grande variabilidade do NDVI no Cerrado decorre da queda das folhas de grande parte dos indivíduos desta formação vegetal, durante o período de seca.

Observa-se, também, que no período seco dos anos 1983, 87, 88 e 91, o NDVI apresentou uma queda acima da média (Figura 2B). Nesses anos, com exceção de 1988, ocorreram eventos de El Niño que ocasionaram diminuição na precipitação na região central do Brasil (Gurgel, 2000). Além disso, em 1988 verificou-se o maior número de focos de fogo já registrados em toda a região central do Brasil (Setzer, comunicação pessoal). De acordo com Tanré et al. (1992) os aerossóis resultantes das queimadas ocasionam redução nos valores do NDVI.

\section{Quarto grupo}

O quarto grupo (azul escuro) da análise de agrupamento representa a caatinga, que está presente em grande parte da região Nordeste do Brasil. Esta vegetação apresenta ciclo anual bem definido, com um período seco marcante (Figura 2C). Os valores mais altos de NDVI se dão entre os meses de abril e maio, que é o final da época das chuvas. Os meses de setembro e outubro apresentam os menores valores de NDVI. Este é o período mais seco da região, com alguns locais apresentando totais mensais de precipitação próximos de zero milímetro, nesta época.

No período de ocorrência do El Niño em 1982/83 e 1991/93, os valores de NDVI ficaram abaixo da média, apresentando uma acentuada queda nos meses mais secos; entretanto, este decréscimo não foi tão acentuado como os que se verificaram na floresta ombrófila densa e no cerrado. Aparentemente, isto ocorre porque, normalmente, grande parte dos indivíduos da caatinga perde as folhas na época seca, fato que também explica a fraca anomalia observada na época seca durante o El Niño de 1986/87. Deve-se destacar que nem todo evento El Niño produz seca na região Nordeste do Brasil, outros fatores são provavelmente mais importantes (Kane, 2000).

\section{Quinto grupo}

Quinto grupo (roxo) representa dois tipos de vegetação: a floresta ombrófila aberta e a floresta ombrófila mista (Figura 1). O primeiro tipo de vegetação se localiza em toda a borda sul da floresta ombrófila densa e o segundo abrange o Estado de Santa Catarina e o sul do Paraná. Esses tipos de vegetação foram classificados, possivelmente, num único grupo, por apresentarem padrões florestais semelhantes, com valores de NDVI bem próximos e ciclo anual comum.

As formações vegetais deste grupo apresentam ciclo anual pouco nítido, como pode ser observado na Figura 2D. O mês de setembro, final do período seco, destaca-se dos demais por apresentar os menores valores de NDVI, enquanto o período em que ocorrem os maiores valores de NDVI não são bem definidos, iniciando-se em novembro e estendendo-se até junho, quando então ocorrem os valores máximos, talvez porque em novembro (início do período chuvoso) há bastante nebulosidade na região, devido à presença da ZCAS, ocasionando diminuição no NDVI; já no outono a nebulosidade diminui, elevando gradualmente o valor do NDVI.

A variabilidade dos tipos de vegetação presentes neste grupo também apresenta oscilações em anos de El Niño (1982/ 83, 1986/87 e, principalmente, 1991/92), porém o El Niño de 1982/83, que foi o mais forte, não causou tanto impacto nesses tipos de vegetação, como nos demais grupos. Observa-se, também, que setembro de 1988 foi o mês que mais se destacou no período analisado, visto que este ano, que não estava associado à ocorrência do El Niño, apresentou, em toda a região central do Brasil, um dos maiores números de focos de fogo já registrados. Como já mencionado, os aerossóis resultantes das queimadas provocaram queda acentuada no NDVI.

\section{Sexto grupo}

O sexto grupo (rosa), é o menor grupo reconhecido pela análise de agrupamento e representa a vegetação das regiões da zona da mata nordestina (litoral leste do Nordeste) e dos campos de Roraima (Figura 1). A cobertura vegetal primitiva da primeira região era de floresta ombrófila densa e aberta e de floresta estacional decidual. Atualmente, esta região está bastante alterada, sendo a agricultura da cana-de-açúcar a principal cobertura vegetal. As vegetações deste grupo apresentam um ciclo anual nítido (Figura 2E). Observa-se que em junho e julho, finais da época chuvosa, ocorrem os maiores NDVI, sendo que os menores valores se situam entre fevereiro e março, dois meses antes do início das chuvas; isto se dá, possivelmente, devido à capacidade de retenção de água pelo solo na região, pois a vegetação consegue atravessar o período seco (outubro a dezembro) sem que ocorra queda marcante no NDVI. Além disso, esta região é influenciada pelos efeitos das brisas marítimas (Kousky, 1981) que possibilitam manter certa umidade, evitando assim uma queda acentuada da folhagem. 
A análise deste grupo também revela que em 1982/83 o NDVI esteve abaixo da média e, em 1991/92, os meses que deveriam ter valores altos de NDVI, apresentaram valores muitos baixos (Figura 2E), sugerindo que, nesses períodos, ocorreu precipitação abaixo do normal. Como conseqüência, os meses (maio a agosto), que deveriam ter valores altos de NDVI no ano seguinte, apresentaram valores abaixo da média. Observa-se, também, que o El Niño de 1987 foi mais ameno que os de 1982/83 e 1991/92. Neste ano, o período de seca não foi tão afetado como nos demais anos de El Niño (Lima, 1991) apresentando, inclusive, NDVI um pouco acima da média; no período chuvoso os valores ficaram ligeiramente abaixo da média. Esses resultados classificou a estação

\section{Sétimo grupo}

O sétimo grupo (vermelho), que representa a região da costa do Oceano Pacífico (Figura 1), não foi analisado, pois não pertence às formações vegetais brasileiras. Ele foi classificado porque a área de estudo da análise de agrupamento compreende áreas da América do Sul, localizadas no entorno do Brasil.

\section{Oitavo grupo}

O oitavo grupo (laranja), aparentemente não representa um grupo fitogeográfico definido; pode-se dizer que é uma área de transição no entorno do cerrado, conforme observado na Figura 1; ele foi classificado devido à reamostragem dos dados, que aumentou a área do pixel de $8 \mathrm{~km}$ para $64 \mathrm{~km}$, o que ocasionou uma "área de confusão" no entorno do cerrado. A vegetação representada neste grupo possui ciclo anual nítido (Figura 2F), e o máximo de NDVI ocorre em maio, que é o final da época chuvosa na maior parte da região, enquanto o mínimo é bem marcante e ocorre em setembro, no final da época seca.

Nesta região, os anos de ocorrência do El Niño (1983, 1987 e 1991/92) apresentaram um período seco bastante pronunciado, principalmente 1992, que teve valores de NDVI bem abaixo da média; entretanto, foi na época da seca do ano de 1988 que ocorreram os menores valores de NDVI. Este ano, como já foi relatado, apresentou o maior número de focos de incêndio, resultando em alta quantidade de fumaça na atmosfera (aerossol) o que, possivelmente, ocasionou esta queda (Tanré et al., 1992).

\section{Nono grupo}

O nono grupo (amarelo), representa as campanhas gaúchas, região de estepe do sul do Brasil (Figura 1). Este tipo de vegetação não apresenta ciclo anual nítido (Figura $2 \mathrm{G}$ ), os valores máximos de NDVI geralmente ocorrem entre março e junho e os valores mínimos, melhor definido, ocorrem no mês de agosto. Nesta região, o período das chuvas é muito variado o que, possivelmente, influencia no padrão do ciclo anual; entretanto, o noroeste do Rio Grande do Sul apresenta um regime de chuvas, conforme sugere a variabilidade do NDVI. Como a maior parte deste grupo se localiza nesta região, este deve ter tido peso maior na caracterização do ciclo anual.

Baixos valores de NDVI são observados em 1982 e 1992 (anos de El Niño), porém isto não deve ter sido ocasionado por seca e, sim, por enchentes, pois a literatura demonstra que há ocorrência do aumento de chuvas para a região sul do Brasil durante o El Niño (Kousky \& Cavalcanti, 1984 e CPTEC, 1998);

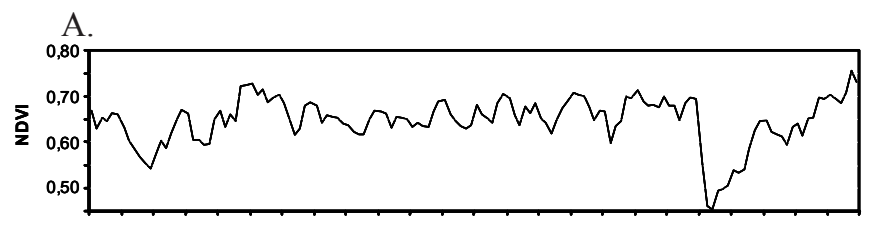

B.

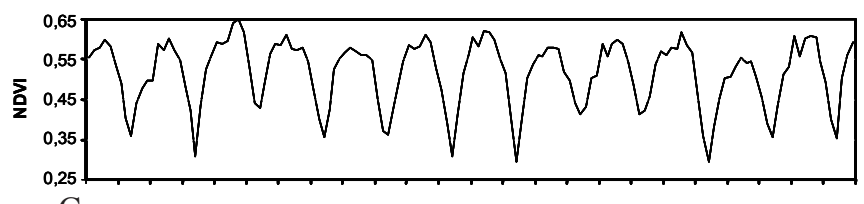

C.

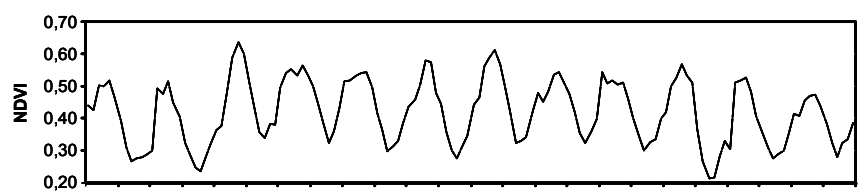

D.
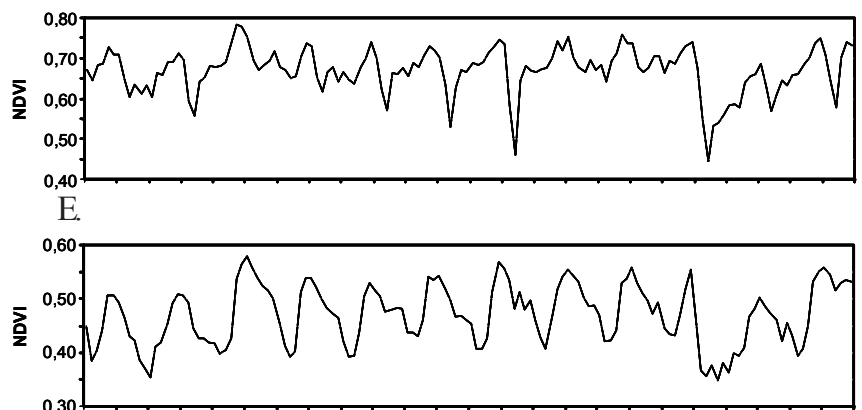

F.

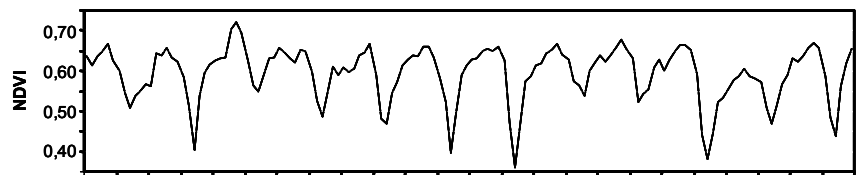

G.

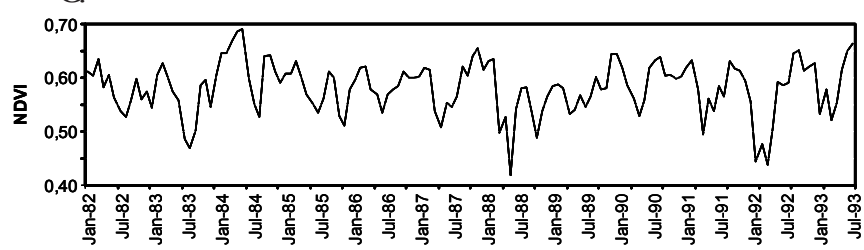

Figura 2. Média mensal da cobertura vegetal obtida através da análise de agrupamento de NDVI: A) segundo grupo, B) terceiro grupo, C) quarto grupo, D) quinto grupo, E) sexto grupo, F) oitavo grupo e G) nono grupo

além disso, logo após o período de queda do NDVI ocorrem valores acima da média, que devem estar relacionados com o aumento de disponibilidade de água no solo. No final de 1987 e início de 1988, também anos de El Niño, não houve queda no NDVI, mas apenas valores máximos de NDVI acima da média, provavelmente porque este evento não foi tão intenso como os demais, o que não ocasionou enchentes muito fortes. No final de 1988, ano de La Niña, caracterizado por pouca chuva no sul, verificou-se forte queda no NDVI.

\section{CONCLUSÕES}

1. A análise de agrupamento realizada nos dados de NDVI, revelou a variabilidade anual e inter-anual e o tempo de 
resposta à precipitação das grandes manchas de vegetação que ocorrem no Brasil.

2. Os tipos de vegetação localizados em regiões com períodos secos e chuvosos marcantes, como é o caso do cerrado e da caatinga, apresentam ciclo anual bem caracterizado e respondem de forma rápida à ocorrência de chuva.

3. Os eventos do ENSO, independente da sua intensidade, afetam distintamente os vários tipos de vegetação.

4. O NDVI das vegetações mais densas e verdes durante todo o ano, como é o caso da floresta amazônica, apresentou maior variabilidade quando ocorreram anomalias climáticas.

5. Nas regiões onde o El Niño provoca diminuição da precipitação e tem um período seco bem definido, como é o caso de grande parte do Nordeste, o NDVI apresentou pouca variabilidade durante o período seco, além de ligeiro decréscimo no período chuvoso.

\section{AGRADECIMENTOS}

Os autores agradecem à Divisão de Sensoriamento Remoto do INPE, por viabilizar a realização deste estudo; o segundo autor agradece ao Conselho Nacional de Desenvolvimento Científico e Tecnológico, Processo número 300486/96-0, pelo suporte. As sugestões dos revisores anônimos contribuíram significativamente para o aprimoramento deste trabalho. Agradecemos também a Claudia Cristina dos Santos e Silvia Pardi Lacruz, pelo apoio.

\section{LITERATURA CITADA}

Almeida, E.S.; Batista, G.T. Índice de vegetação versus precipitação na Amazônia. In: Simpósio Brasileiro de Sensoriamento Remoto, 1998, Santos. Anais... Santos. Instituto Nacional de Pesquisas Espaciais, 1998, CD-Rom

Anyamba; A.; Eastman, J.R. Interannual variability of NDVI over Africa and its relation to El Niño/Southern Oscillation. International Journal of Remote Sensing, London, v.13, p.2533-2548, 1996.

Barbosa, H.A. Análise espaço temporal de índice de vegetação AVHRR/NOAA e precipitação na região nordeste do Brasil, em 1982-85. São José dos Campos: INPE, 1998. 164p. Dissertação Mestrado

Batista, G.T.; Shimabukuro, Y.E.; Lawrence, W.T. Monitoramento da cobertura florestal através de índice de vegetação do NOAA-AVHRR. In: Simpósio Brasileiro de Sensoriamento Remoto, 7, 1993, Curitiba. Anais... São José dos Campos: Instituto Nacional de Pesquisas Espaciais, 1993. p.30-37.

Batista, G.T.; Shimabukuro, Y.E.; Lawrence, W.T. The long-term monitoring of vegetation cover in the Amazonian region of northern Brazil using NOAA-AVHRR data. International Journal of Remote Sensing, London, v. 18, n.15, p.3195-3210, 1997.

CPTEC - Centro de Previsão de Tempo e Estudos Climáticos. Conseqüências do fenômeno El Niño sobre o território brasileiro e perspectivas para 1998. www.cptec.inpe.br/ products/elninho. Jan. 1998.
Eastman, J.R.; Fulk, M. Long sequence time series evaluation using standardized principal components. Photogrammetric Engineering \& Remote Sensing, Bethesda, v.59, n.8, p.1307$1312,1993$.

Goddard Space Flight Center's Distributed Active Archive Center (DAAC). http://daac.gsfc.nasa.gov/CAMPAIGN_DOCS/ LAND_BIO/GLBDST_News.html. Jan. 1999.

Gurgel, H.C. Variabilidade espacial e temporal do NDVI sobre o Brasil e suas conexões com o clima. São José dos Campos: INPE, 2000. 114p. Dissertação Mestrado

IBGE - Instituto Brasileiro de Geografia e Estatística. Recursos naturais e meio ambiente: uma visão do Brasil. Cladeiron, S. S. (coorden). Rio de Janeiro: IBGE, Departamento de Recursos Naturais e Estudos Ambientais, 1993, 154p.

Kane, R. P. Relationship between El Niño timings and rainfall extremes in NE Brazil, São Paulo and South Brazil. Revista Brasileira de Meteorologia, São José dos Campos, v.15, n.1, p.45-57, 2000.

Kousky, V.E. Diurnal rainfall variation in Northeast Brazil. Monthly Weather Review, Boston, v.108, n.4, p.488-498, 1980.

Kousky, V.E; Cavalcanti, I.F.A. Eventos oscilação - El Niño: características, evolução e anomalias de precipitação. Ciência e Cultura, São Paulo, v.36, n.11, p.1888-1899, 1984.

Lima, M.C. Variabilidade da precipitação no litoral leste da Região Nordeste do Brasil. São José dos Campos: Instituto Nacional de Pesquisas Espaciais, 1991. 192p. Dissertação Mestrado

Liu, W.T.; Massambani, O.; Festa, M. Normalized difference vegetation index for the South American continent used as a climatic variability indicator. In: International Symposium on Remote Sensing of Environment, 24, 1991, Rio de Janeiro. Proceedings... Ann Arbor: University of Michigan, 1991, p.725-732.

Potter, C.S.; Brooks, V. Global analysis of empirical relations between annual climate and seasonality of NDVI. International Journal of Remote Sensing, London, v.19, n.15, p.2921-2948, 1998.

Rao, V.B.; Hada, K. Characteristics of rainfall over Brazil: Annual variations and connections with the Southern Oscillation. Theoretical and Applied Climatology, New York, v.42, n.2, p.81-91, 1990.

Richards, J.A. Remote sensing digital images analysis: an introduction. Berlin: Springer-Verlag, 1986, 281p.

Richard, Y.; Poccard, I. A statistical study of NDVI sensitivity to seasonal and interannual rainfall variations in Southern Africa. International Journal of Remote Sensing, London, v.19, n.15, p.2907-2920, 1998.

SAS. SAS procedure guide, release 6.03. Cary: SAS Institute Inc., 1988. 441p.

Schultz, P.A.; Halpert, M.S. Global analysis of the relationships among a vegetation index, precipitation and land surface temperature. International Journal of Remote Sensing, London, v.16, n.15, p.2755-2777, 1995.

Tanré, D., Holben, B.N., Kaufman, Y.J. Atmospheric correction algorithm for NOAA-AVHRR products: Theory and application. IEEE Transactions on Geoscience and Remote Sensing, Picataway, v.30, n.2, p.231-248, 1992. 